\title{
Two-dimensional transport and wall effects in the thermal diffusion cloud chamber. II. Stability of operation.
}

Anne Bertelsmann

Richard H. Heist

Fairfield University, rheist@fairfield.edu

Follow this and additional works at: https://digitalcommons.fairfield.edu/engineering-facultypubs (c) 1997 American Institute of Physics.

The final publisher PDF has been archived here with permission from the copyright holder. https://aip.scitation.org/doi/abs/10.1063/1.473401

\section{Peer Reviewed}

\section{Repository Citation}

Bertelsmann, Anne and Heist, Richard H., "Two-dimensional transport and wall effects in the thermal diffusion cloud chamber. II. Stability of operation." (1997). Engineering Faculty Publications. 158. https://digitalcommons.fairfield.edu/engineering-facultypubs/158

\section{Published Citation}

Bertelsmann, A., \& Heist, R. H. (1997). Two-dimensional transport and wall effects in the thermal diffusion cloud chamber. II. Stability of operation. The Journal of chemical physics, 106(2), 624-634. https://doi.org/10.1063/ 1.473401 .

This item has been accepted for inclusion in DigitalCommons@Fairfield by an authorized administrator of DigitalCommons@Fairfield. It is brought to you by DigitalCommons@Fairfield with permission from the rightsholder(s) and is protected by copyright and/or related rights. You are free to use this item in any way that is permitted by the copyright and related rights legislation that applies to your use. For other uses, you need to obtain permission from the rights-holder(s) directly, unless additional rights are indicated by a Creative Commons license in the record and/or on the work itself. For more information, please contact digitalcommons@fairfield.edu. 


\title{
Two-dimensional transport and wall effects in the thermal diffusion cloud chamber. II. Stability of operation
}

\author{
Anne Bertelsmann and Richard H. Heist ${ }^{\mathrm{a})}$ \\ Department of Chemical Engineering, University of Rochester, Rochester, New York 14627-0166
}

(Received 17 June 1996; accepted 2 October 1996)

\begin{abstract}
In this paper, the second of a series of two presenting a detailed description of thermal diffusion cloud chamber operation, we address the operational stability of the vapor-gas mixture in a diffusion cloud chamber with respect to density profile extrema and the accompanying possibility of buoyancy-driven convective flow disturbances. We examine conditions for stable operation (no convective flow disturbances) in the central portion of the cloud chamber, as well as conditions necessary for stable operation in the vicinity of the cloud chamber wall. We find that the total density profile in the central portion of the cloud chamber can pass through a density minimum even though the density at the upper plate surface is less than the density at the lower plate surface. This local density profile inversion can result in unstable (convective) behavior that propagates through the cloud chamber. Furthermore, we find that local extrema in the total density profile near the chamber wall can lead to subtle, convective flows that are difficult to detect yet can exert a profound influence on nucleation in the central portion of the cloud chamber. We have developed a simple method to estimate the limiting total pressure in a cloud chamber that will support stable operation. From results of our investigations based upon this method, it appears that the thermal diffusion cloud chamber is best suited for experiments at higher temperatures where the accessible total pressure range is largest. Finally, we find that results of our investigation into the effects of total pressure and kind of background gas on nucleation in diffusion cloud chambers involving the low molecular weight alcohols and hydrogen and helium background gases cannot be explained on the basis of these kind of density disturbances occurring within the diffusion cloud chamber. Also, for (relatively) low vapor pressure materials, such as 1-pentanol or other high molecular weight alcohols and alkanes stability limitations may preclude nucleation measurements at low temperatures using a diffusion cloud chamber altogether. (c) 1997 American Institute of Physics. [S0021-9606(97)51602-3]
\end{abstract}

\section{INTRODUCTION}

In the preceding paper, ${ }^{1}$ hereafter referred to as $\mathrm{I}$, we reported results of a two-dimensional $(z, r)$ analysis of transport processes occurring during operation of a thermal diffusion cloud chamber (TDCC). We discussed the solution of the two-dimensional mass and energy balances and illustrated various solutions with representative plots of supersaturation and nucleation rate profiles. Through the use of appropriate boundary conditions, we described chamber operation with a wet and a dry cloud chamber wall. We investigated the influence of the aspect (diameter to height) ratio, the conditions at the cloud chamber wall, and overheating of the wall on these supersaturation and nucleation rate profiles. We confirmed, in a quantitative fashion, that conditions at the (wet or dry) wall do not seriously affect conditions at the center of the chamber, provided the aspect ratio is sufficiently large and the wall is not significantly overheated; and, importantly, we provided criteria allowing us to specify that an aspect ratio of 7.5 or greater was necessary in order to make quantitative nucleation rate measurements. However, even for this aspect ratio, care must be taken in the analysis of experimental nucleation rate data since the counting area being used can adversely affect the value of the measured

\footnotetext{
a) Author to whom all correspondence should be addressed.
}

nucleation rate. In I, we found that for an aspect ratio of 5 , the nucleation rate in the center of the chamber is significantly reduced due to the influence of the chamber wall. Furthermore, we found that the effect of a wet wall, where the vapor near the wall is depleted, on the chamber supersaturation profile, is nearly identical to that of a dry wall, where the temperature near the wall is elevated.

One important issue, which was not addressed in $\mathrm{I}$, is the stability of the vapor-gas mixture in the TDCC with respect to buoyancy-driven convective motion. We note that use of the terms stable (stability) and unstable (instability) in this paper refer to conditions resulting from having the total density gradient throughout the cloud chamber either decreasing monotonically with height (stable) or passing through an extrema at some point within the cloud chamber, thus creating conditions that support buoyancy-driven convective flow (unstable). We already know that elevated total pressures or the use of heavier background gases in the TDCC may lead to convection and copious nucleation. ${ }^{2}$ Also, a dependence of nucleation rate on wall heat (temperature) has been reported elsewhere. ${ }^{3,4}$ In I, we showed that this behavior cannot be explained by the effect of the presence of the dry, overheated wall on the radial supersaturation and nucleation rate profiles. $^{1}$

In this paper we discuss specifically the stability of the 
vapor-gas mixture in the diffusion cloud chamber with respect to convection. An inversion of the total density gradient in the central portion of the TDCC leads to convective flow and copious nucleation, a condition that can be predicted and detected visually. ${ }^{1,2,5}$ Note that even though the density at the top plate is less than the density at the lower plate (a seemingly stable configuration), the density profile can still be unstable if it passes through a local minimum (a local inversion) at some location between the upper and lower plates.

An inversion of the density gradient near the wall can also lead to convective motion, but in a much more subtle form. This (subtle) convective motion can affect the conditions in the nucleation zone and thus influence nucleation measurements; however, it usually cannot be detected visually. Since the presence of the wall is not considered in the (commonly used) one-dimensional analysis of TDCC operating conditions, the existence of these density minima is usually overlooked or treated on an entirely empirical, ad hoc basis. ${ }^{4}$ We have developed a general, semiquantitative method that allows one to predict the operating conditions under which a density inversion near the wall (wet or dry) is expected and can thus determine, in an a priori fashion, the allowable temperature and pressure regions for proper, reliable TDCC operation. We describe this procedure in this paper, and we apply it to systems studied in our laboratory and discuss our data with respect to stability of the vaporgas mixture in the high-pressure diffusion cloud chamber (HPCC).

\section{MATHEMATICAL DESCRIPTION}

The derivation for the two-dimensional equations for the mass and energy transport in the diffusion cloud chamber has been presented in I and will not be repeated here. If we assume no chemical reaction, no external forces, no net pressure gradient, and ideal gas behavior, and we neglect thermodiffusion effects, the two-dimensional mass balance in cylindrical coordinated reduces to the following expression: ${ }^{1}$

$$
\begin{array}{r}
\left(\frac{1}{r}+b \frac{\partial \ln T}{\partial r}\right) \frac{\partial \ln (1-x)}{\partial r}+\frac{\partial^{2} \ln (1-x)}{\partial r^{2}} \\
b \frac{\partial \ln T}{\partial z} \frac{\partial \ln (1-x)}{\partial z}+\frac{\partial^{2} \ln (1-x)}{\partial z^{2}}=0
\end{array}
$$

With the same assumptions, the two-dimensional energy balance reduces to the following expression: ${ }^{1}$

$$
\begin{aligned}
\left(\frac{1}{r}+\right. & \left.\frac{\partial \ln \lambda}{\partial r}-\frac{c D_{A B} c_{p}}{\lambda} \frac{\partial \ln (1-x)}{\partial r}\right) \frac{\partial T}{\partial r}+\frac{\partial^{2} T}{\partial r^{2}} \\
& +\left(\frac{\partial \ln \lambda}{\partial z}-\frac{c D_{A B} c_{p}}{\lambda} \frac{\partial \ln (1-x)}{\partial z}\right) \frac{\partial T}{\partial z}+\frac{\partial^{2} T}{\partial z^{2}}=0
\end{aligned}
$$

These two balance equations are solved together with appropriate boundary conditions to determine the two-dimensional mole fraction and temperature profiles in the cloud chamber. ${ }^{1}$ Once these profiles are known, the supersaturation, nucleation rate, and density profiles can then be computed. ${ }^{1,5}$

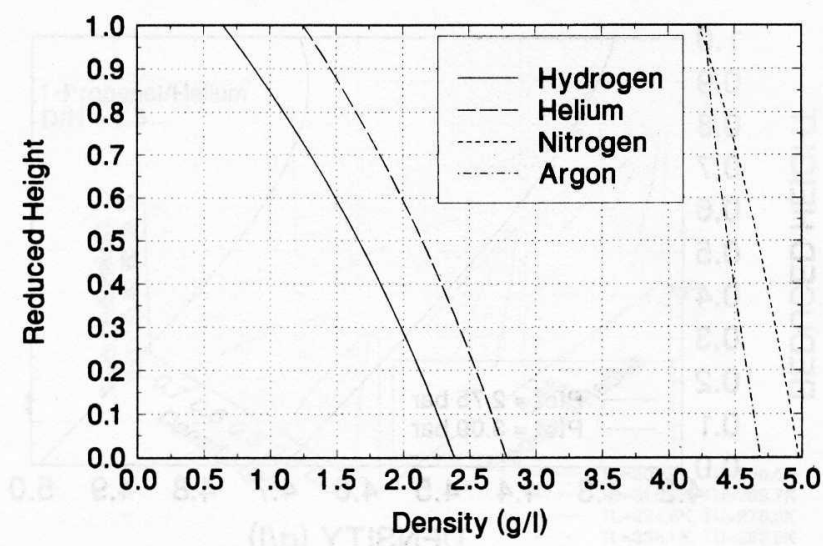

FIG. 1. Computed density profiles in the center of the TDCC for experiments using 1-butanol and four different background gases.

\section{DENSITY PROFILES IN THE CENTER OF THE TDCC}

An important consideration for the operation of an upward diffusion cloud chamber is the overall stability with respect to buoyancy-driven convection of the vapor-gas mixture in the chamber. For a stable layering, the total density must decrease monotonically with height from the lower plate surface to the upper plate surface. The density gradient of the background gas by itself is always unstable (increases with height), since it is heavier at the cooler, upper plate and lighter at the warmer, lower plate. The presence of the condensable vapor actually stabilizes the overall density gradient (with respect to buoyancy-driven convective motion), since the vapor pressure is higher at the lower plate and lower at the upper plate. Instability (giving rise to convective motion) can occur when the background gas dominates the total density gradient. This can occur at elevated total pressures, when a higher molecular weight background gas is used, or at low temperatures (and lower total pressures), where the vapor pressure of the condensable is small. An inversion in the density gradient in the center region of the TDCC can lead to convection and often produces copious nucleation. ${ }^{2,5}$ The presence of convection can often be detected visually, since the nucleated droplets tend to fall with curved trajectories. Under these conditions the mass and energy balance equations no longer accurately describe the transport processes occurring inside the chamber and reliable nucleation measurements cannot be made.

Figure 1 shows density profiles computed for the central portion of the HPCC using Eqs. (1) and (2) and assuming an infinite aspect ratio, thus decoupling wall effects from the transport in the central portion of the chamber. These profiles are based upon data obtained from actual experiments in the HPCC involving 1-butanol in hydrogen, helium, nitrogen, and argon background gases. In all cases the nucleation temperature was approximately $334 \mathrm{~K}$. Also, the temperature, supersaturation, and nucleation rate profiles in the central portion of the HPCC for these experiments are given in I as Figs. 14, 15, and 16, respectively. For the hydrogen and helium experiments $\left(P_{\text {tot }}=7.5 \mathrm{bar}\right)$, the total density profiles clearly decrease with height. For the nitrogen experiment 


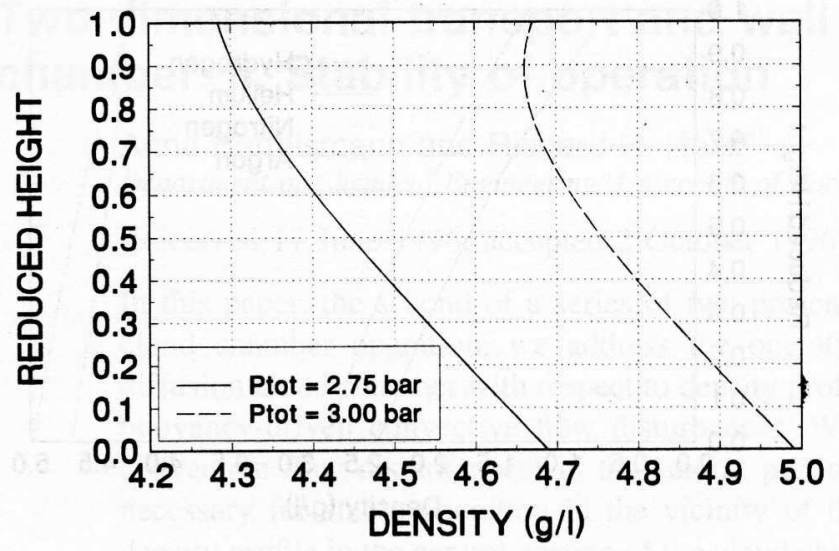

FIG. 2. Representative density profiles in the center of the TDCC for experiments using 1-butanol in argon at total pressures of 2.8 and 3.0 bar.

$\left(P_{\text {tot }}=3.9 \mathrm{bar}\right.$ ), the decrease with height is less than for the lighter gases, but the vapor-gas mixture is still stable. However, for the argon experiment $\left(P_{\text {tot }}=2.8 \mathrm{bar}\right)$, the density gradient in the upper portion of the chamber is nearly vertical. A slight disturbance, such as a small increase in total pressure, could result in a local minimum of the density near the upper plate, which could then lead to convective motion, even though the density at the bottom plate is greater than that at the top plate. We find this predicted behavior agrees well with our experimental observations. For example, the data used in this analysis (for Fig. 1) were taken from one of our experimental investigations, during which we observed that an increase in pressure of just 0.2 bar (over the $2.8 \mathrm{bar}$ ) led to an unstable density gradient predicted for the central portion of the HPCC and was accompanied by (observed) copious nucleation. Reducing the total pressure by $0.2 \mathrm{bar}$, restored quiescent behavior. Fig. 2 shows the (onedimensional case) density profile for 1-butanol in argon at $P_{\text {tot }}=2.8$ bar and $P_{\text {tot }}=3.0$ bar. At total pressures above 2.8 bar, convective motion and copious nucleation were observed in the chamber. It is important to note that the total density at the lower plate is still larger than the total density at the upper plate and that the total density profile for a total pressure of 3.0 bar exhibits a minimum near the upper plate. This local inversion appears to cause buoyancy-driven convection, which propagates through the chamber and transports additional (perhaps warmer) material into the nucleation zone, resulting in the observed increase in the nucleation rate. We also observed that the trajectories of the falling drops in this experiment were no longer vertical.

\section{DENSITY PROFILES NEAR THE WALL OF THE TDCC}

For proper operation of a TDCC, it is important to consider not only the stability of the vapor-gas mixture in the central region of the chamber (which can be obtained using a one-dimensional analysis), but also at the wall. Our twodimensional analysis of TDCC operation [solution of Eqs. (1) and (2) with a finite aspect ratio] suggests that undesirable variations in total density can occur in the vicinity of the chamber wall at pressures well below those for which the total density profile in the central portion of the cloud chamber becomes unstable. These variations could result in convective flow that would disrupt proper chamber operation in rather subtle fashions. Although it is difficult to predict the detailed structure of convective flow that could result from inversions in the total density profile near the chamber wall, one can easily imagine a kind of convective flow pattern in which an inversion in the local density would lead to a slow upward flow of vapor-gas mixture along the wall to the region adjacent to the upper plate. This flow could then continue along the region parallel to the surface of the upper (cooler) plate to the center region and turn downward in the central region of the chamber toward the lower plate as it encounters a similar flow from the other (symmetric) side of the cloud chamber. As the flow moves, it passes through the nucleation zone to the region adjacent to the lower plate surface, where it could make its way back to the region adjacent to the wall and slowly move back up the wall, competing a large convection cell. This type of flow could be slow (and subtle) and would not appear to grossly affect the overall operation of the chamber, i.e. no copious nucleation or droplets falling with curved tracks, so it would not be detected visually. Furthermore, the flow, as it comes from the region adjacent to the upper plate and passes through the nucleation region, could be imagined to contain a lesser amount of condensable (and possibly be at a lower temperature) and would thus contribute to a lowering of the nucleation rate. This type of instability would explain reported experimental observations in which the nucleation rate was observed to decrease with increases in wall heat (temperature) ${ }^{3,4}$ As we discuss below, wall overheating leads to increasingly larger density disturbances in the region adjacent to the chamber wall. We note that one could also easily imagine that this kind of large convective cell could also flow in the opposite direction from that just described if the initial conditions at the wall were such as to promote a falling flow rather than a rising flow. Again, based upon the analysis given here, it is difficult to predict the nature or structure of any resulting convective flow. We are, however, able to predict the existence of conditions that will give rise to convective flow because of the resulting buoyancy driving forces.

These types of observations, i.e. the nucleation rate depending upon wall temperature, have been made in our laboratory and by others, and in most cases the TDCC appears to be functioning properly. This is the real danger associated with this kind of behavior. During these experiments, there is no obvious warning of any difficulty (unless one carries out extensive, empirical wall temperature versus nucleation measurements for each investigation), and the nucleation measurements are generally reproducible. This gives the investigator the (false) sense that the nucleation measurement is reliable and the cloud chamber is functioning properly. Furthermore, density profile inversions occur at the chamber wall before corresponding density inversions occur in the central portion of the cloud chamber. This is why it is essential that a complete two-dimensional analysis of chamber operation be utilized in all nucleation investigations purport- 


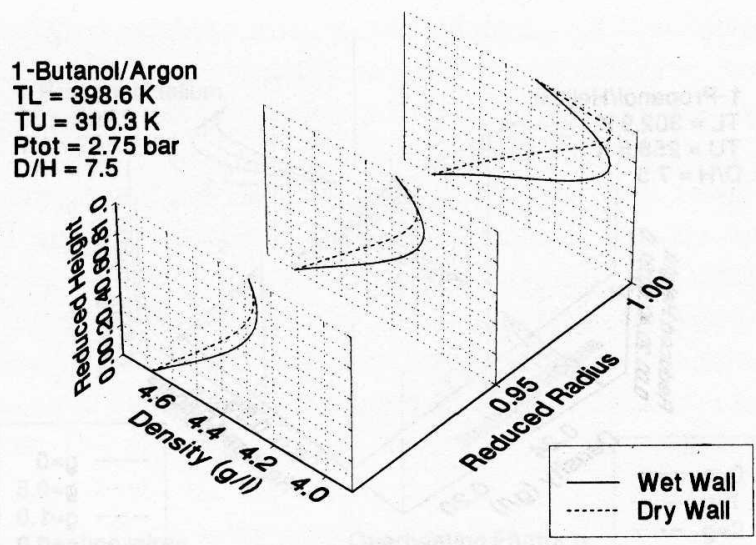

FIG. 3. Computed density profiles in the vicinity of the chamber wall for experiments using 1-butanol in argon for a wet and a dry chamber wall.

ing to be quantitative in nature. Consequently, it is important to be able to predict, in a convenient, reliable, and a priori fashion, the density gradient near the wall under all experimental conditions of varying total pressures, different temperature ranges, different background gases, and ranges of wall temperature before each nucleation measurement to determine if this kind of convective flow may be expected.

\section{Comparison of wet and dry walls}

Because of the difference in wall (boundary) conditions the density profile near the wall differs for wet and dry walls. ${ }^{1}$ Figure 3 shows the density profiles for 1 -butanol in argon at reduced radii of $0.9,0.95$, and 1.0 (1.0 being directly at the wall). The system 1-butanol in argon was chosen for this discussion because it exhibits the largest density effects and thus most clearly illustrates the difference between a wet and a dry chamber wall. As can be seen in Fig. 3, the density profile for both the wet and the dry wall exhibits a minimum in the density in the vicinity of the wall. In both cases, the value of the density is similar, but the location of the minimum differs. For the dry wall the minimum occurs close to the upper plate; for the wet wall the minimum occurs closer to midway between the upper and lower plates. In the case of a wet wall, the minimum in the density profile is caused by the depletion of the (heavier) vapor close to the wall. This depletion is strongest halfway between the two plates, where the difference between the actual and the equilibrium mole fraction is largest. For both the wet and dry wall cases, the minimum in the density profiles propagates into the chamber. It is still detectable at a reduces radius of 0.90. Under these conditions a buoyancy-driven flow, perhaps similar to those described above, is possible. Density minima that occur only in the immediate vicinity of the wall and are damped out before they propagate toward the center of the chamber may be less likely to result in flow, because of the proximity of the wall.

The temperature range, the total pressure, the type of background gas being used, and, in the case of a dry wall, the overheating of the wall, are all factors that significantly influence the extent of these density minima. We discuss these

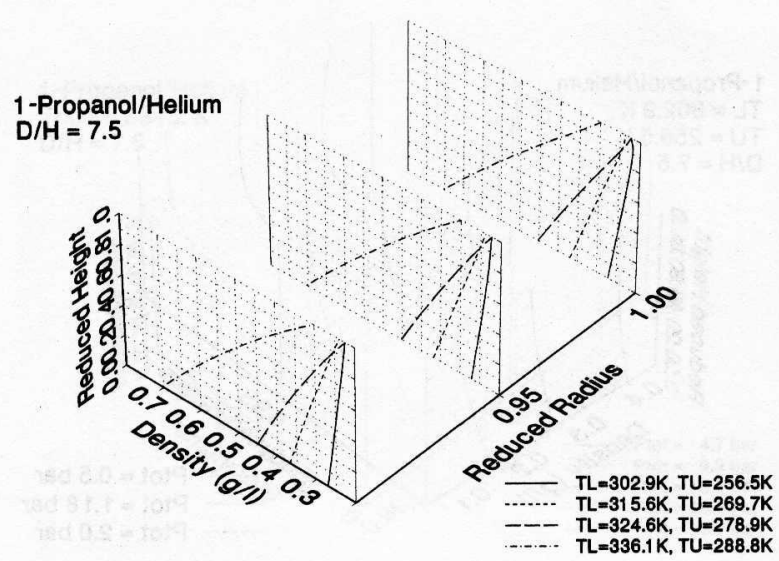

FIG. 4. Computed density profiles in the vicinity of the dry chamber wall for experiments of 1-propanol in helium at different upper and lower plate temperatures.

influences individually in the following sections. We illustrate their effects for either a wet or a dry wall case since the resulting effect on the depth and extent of the density minimum is qualitatively the same, although the position of the density minimum along the wall differs somewhat for the two cases.

\section{Effect of plate temperatures}

While the temperatures of the lower and upper plates do not affect the radial nucleation rate profile significantly, ${ }^{1}$ they do have an important influence on the total density profile near the wall. Figure 4 shows computed density profiles near the dry wall for 1-propanol in helium for four different upper and lower plate temperature ranges. The total pressure for these experiments varied between 1.2 and 1.6 bar. For the lowest temperature range, the density profile is nearly vertical and exhibits a minimum at the wall. For the higher temperature ranges, the density profile decreases more strongly with height, resulting in a stable layering of the vapor-gas mixture all the way to the wall. Thus, higher temperatures appear to be favorable in preventing convective flow of this type. The same result is observed for the wet wall case.

\section{Total pressure effect}

In I, we showed that the effect of total pressure on the radial supersaturation and nucleation rate profiles is small; ${ }^{1}$ however, the total pressure does have an effect on the density profile. Figure 5 shows the density profile near a wet wall for 1-propanol in helium at three different pressures. The temperature ranges for these three calculations are identical. At the higher total pressure (2.0 bar in these calculations), the density gradient clearly exhibits a minimum at the wall and propagates into the chamber-it can still be detected at a reduced radius of 0.95 . At lower total pressures the minimum becomes less pronounced, e.g. for 0.5 bar the density profile is stable all the way to the wall. Thus, an increase in total pressure has an unfavorable influence on the density profile. 


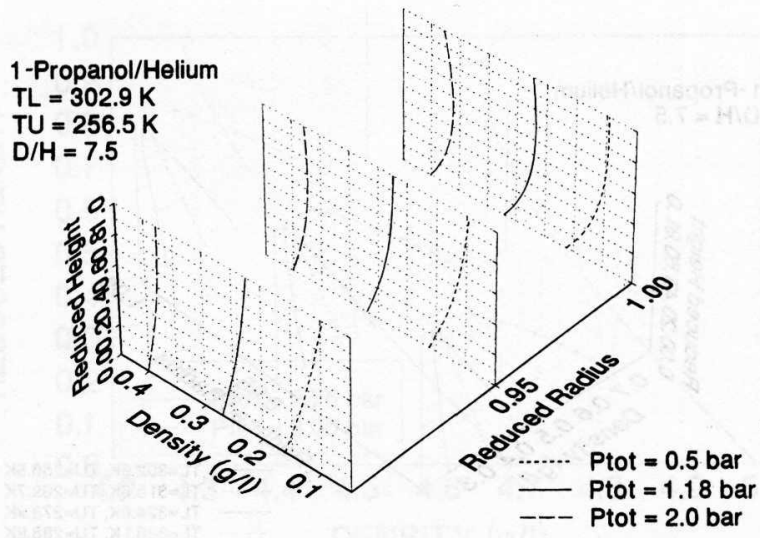

FIG. 5. Computed density profiles in the vicinity of the wet chamber wall for experiments of 1-propanol in helium at different total pressures.

Convective disturbances could be more likely at higher pressures, particularly at lower temperatures. These same observations apply to the dry wall case.

\section{Effect of different background gases}

Figure 6 illustrates the effect of different background gases on the density profile near the wet wall for actual experiments we carried out with 1-butanol in the HPCC. The total pressure for our experiments using 1-butanol in hydrogen and in helium is 7.5 bar, using nitrogen it was 3.9 bar and using argon it is 2.8 bar. Recall that the density profiles at the center of the HPCC for these particular experiments were discussed in I. Figure 6 illustrates that a minimum in the density occurs near the wall, and it is most pronounced for the argon and nitrogen background gases while it is virtually nonexistent for the helium and hydrogen background gases. This is consistent with our observations in the total pressure investigation just described. Elevated total pressure and heavier background gases both result in a stronger influence of the background gas on the total density profile. Furthermore, under these conditions the density profile slightly



FIG. 6. Computed density profiles in the vicinity of the wet chamber wall for experiments of 1-butanol in four different carrier gases.

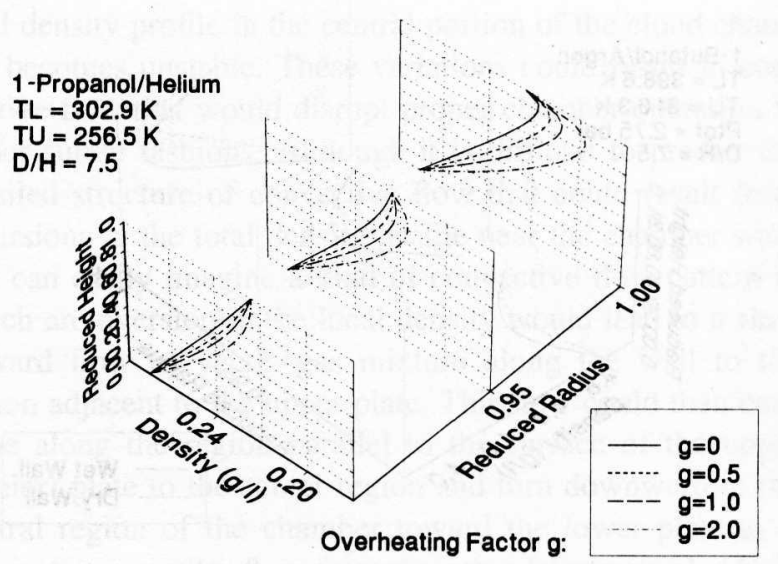

FIG. 7. Computed density profiles in the vicinity of the evenly overheated dry chamber wall for experiments of 1-propanol in helium. $g$ represents an overheating factor and determines the degree of overheat. The case of $g=0$ corresponds to no overheating.

removed from the wall becomes closer to vertical and thus more susceptible to disturbances at the wall, e.g. wall temperature.

\section{Effect of overheating the chamber wall}

In the case of a dry chamber wall, heating the wall can cause local density minima near the wall. Figure 7 shows the density profiles near the chamber wall for different degrees of even overheating for data from our experiment with 1-propanol in helium. At the wall all these density profiles exhibit minimum close to the upper plate. These minima become more pronounced for higher degrees of overheat. At a reduced radius of 0.95 the density profiles for higher degrees of overheat continue to exhibit minima. The higher the degree of overheat, the more pronounced is the minimum and the further it propagates into the chamber. This is consistent with observations from our laboratory, as well as from other laboratories, in which it has been found that nucleation in the interior portion of the TDCC depends upon the power being supplied to the heater wires (thus affecting the wall temperature) used to keep the chamber wall clear of condensate. Depending upon the structure of the resulting convective flow cells, this disturbance could result in either a decrease in nucleation or an increase in nucleation in the interior porting of the TDCC as the power to the wall heater wires is varied. A further indication that these observed dependencies of nucleation in the interior portion of the TDCC on wall heater power is due to this sort of (subtle) convective flow has been given in I in which we discussed the effect of overheating on the calculated radial nucleation rate and supersaturation profiles. ${ }^{1}$ There it was shown that the center region of the chamber is not significantly affected by moderate increases in wall overheating. These simulations did not account for the existence of a buoyancy-driven convective flow.

We have also examined the effect of using heating wires (uneven heating) on the total density profile. Since the chamber walls are usually heated by two to four heating wires, the 


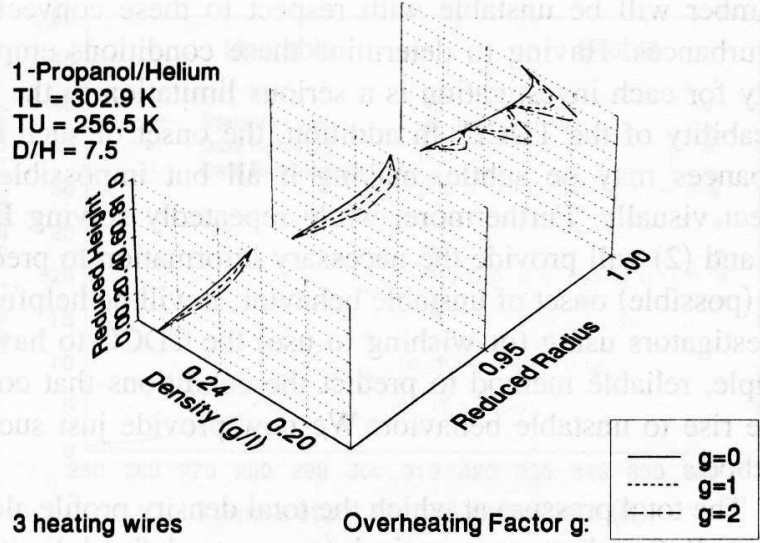

FIG. 8. Computed density profiles in the vicinity of the unevenly overheated dry chamber wall for experiments of 1-propanol in helium.

temperature profile at the wall actually exhibits maxima at the location of the heating wires. This leads to overheating at these positions, since the wall temperature between the heating wires has to be maintained high enough to prevent condensation. We examined this behavior using a periodic temperature profile. Figure 8 shows the density profile near the wall for data from an experiment with 1-propanol in helium. Examples of even overheating and overheating by two, three, and four heating wires are shown. The density profiles at the wall clearly exhibit local extrema due to the periodic temperature profile. We note that these oscillations damp out rapidly as we probe further into the chamber. At a reduced radius of 0.95 the profiles for two, three, and four heating wires are nearly indistinguishable. The resulting average density profile at that reduced radius, however, may continue to exhibit a minimum if the degree of overheat is large. It appears that variations in total density created by the use of discrete heating wires do not have a significant influence on the vapor-gas stability near the wall, but the overheating that occurs due to the use of these discrete heating wires can lead to instability and thus severely influence nucleation measurements made with a TDCC.

\section{COMPARISON WITH EXPERIMENTAL RESULTS}

The behavior of the vapor-gas density gradient near the wall we report here can significantly affect operation of the diffusion cloud chamber. The existence of a convective flow that transports either added or less vapor at different temperatures into the nucleation zone can manifest itself as a varying (with, say, wall heater power) nucleation rate. In order to compensate, the temperature difference between the two plates must be changed to modify the actual supersaturation in the nucleation zone. However, this modified supersaturation is not driven by the nucleation process itself, but rather by the operation of the cloud chamber. Furthermore, the temperature and supersaturation profiles calculated using the (one- or two-dimensional) mass and energy balance equations no longer accurately reflect these profiles in the chamber, since these equations do not account for this type of transport. When interpreting data obtained using a TDCC

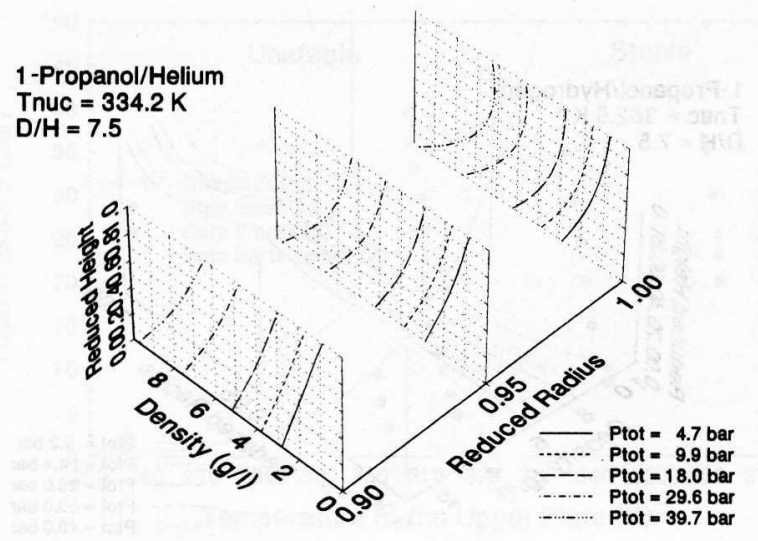

FIG. 9. Computed density profiles in the vicinity of the wet chamber wall for a series of experiments using 1-propanol in helium, where the total pressure was varied at a constant nucleation temperature of $334.2 \mathrm{~K}$.

(or a HPCC), it is nearly impossible to distinguish empirically between effects that are inherent to the nucleation process and effects that are an artifact of the TDCC unless these latter effects are accompanied by pathologic behavior, i.e. copious nucleation.

In order to eliminate the possibility of density-driven instabilities, TDCC operating conditions must be such that the total density profile is stable at every point of the chamber. Under these conditions, buoyancy-driven transport cannot develop. Recall, for example, from the discussion given above that our reported total pressure effects come from experiments that appear to be consistent with conditions favoring these density minima. ${ }^{2,5,6}$ We report that critical supersaturation increases with increasing total pressure; that the effect is more pronounced at lower temperatures; and that the effect is more pronounced for heavier background gases. As we have just shown, these density minima are favored at low temperatures, elevated pressures, and with heavier background gases. We also report that the background gas effects appear to increase with the molecular weight of the alcohol vapors we have examined. We note that the higher the molecular weight of the alcohols in the homologous series, the lower their vapor pressure at a given temperature. Thus there is less condensable in the chamber near the wall, making density extrema more likely to develop. As a result, it could be suggested that our reported total pressure effects should not be attributed to the nucleation process but rather to artifacts of HPCC design and operation. The considerations resulting from our analysis (in I) require that nucleation data gathered using the HPCC (or a TDCC, in general) be examined carefully in the context of the solutions to our twodimensional equations describing transport in the cloud chamber, i.e. solutions to Eqs. (1) and (2). We now examine carefully our HPCC data in this context.

Figure 9 shows the density profiles near the wall for a series of experiments involving 1-propanol in helium at a nucleation temperature of $334.2 \mathrm{~K}$ and over a total pressure range from (approximately) 5 to 40 bar. The density profiles for 4.7 and 9.9 decrease monotonically from the lower to the 


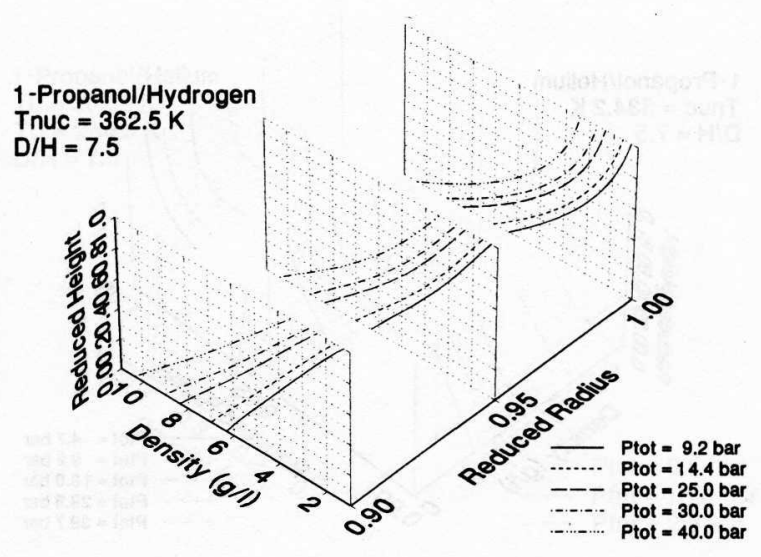

FIG. 10. Computed density profiles in the vicinity of the wet chamber wall for a series of experiments using 1-propanol in hydrogen, where the total pressure was varied at a constant nucleation temperature of $362.5 \mathrm{~K}$.

upper plate surfaces throughout the HPCC. Under these conditions a convective flow or convection cells cannot develop. At a total pressure of $18 \mathrm{bar}$, the density profile along the chamber wall approaches vertical. Only at the elevated pressures of 29.6 and 39.7 bar do the density profiles at the wall shown in Fig. 9 exhibit a minimum. Notice in Fig. 9 that the development of a density minimum is first indicated by a vertical density gradient at the upper plate. Thus, it appears from this analysis that a convective flow of some sort could have developed only for those experiments carried out at the highest of our total pressures.

We recall at this point that using lighter background gases (i.e., hydrogen) favor stability in the cloud chamber. We also recall that operation in higher temperature ranges (i.e., corresponding to our experiments with nucleation temperatures of $348 \mathrm{~K}$ and above) also favors stability. Using this same analysis, we have examined all our alcohol critical supersaturation versus total pressure data (i.e., methanol, ethanol, 1-propanol, and 2-propanol) obtained using the HPCC and hydrogen as a background gas and have verified that the total density profile throughout the HPCC was stable throughout the entire pressure range at all the reported nucleation temperatures. This behavior is illustrated in Fig. 10, where we show representative total density profiles near the wall for experiments involving 1-propanol in hydrogen at a nucleation temperature of $363 \mathrm{~K}$. In this case the total density profile decreases monotonically throughout the entire HPCC, even at a total pressure of $40 \mathrm{bar}$. The results of the total pressure experiments using hydrogen as a background gas were not affected by convective disturbances. A similar analysis of our experiments using helium as a background gas in the higher nucleation temperature ranges (i.e., for nucleation temperatures above approximately $348 \mathrm{~K}$ ) clearly illustrated that in all cases the total density profile throughout the HPCC did not exhibit extrema so that these data, as well were not affected by convective disturbances.

\section{Stability limit for cloud chamber operation}

It is desirable to be able to predict, a priori, the operating conditions for any TDCC (or HPCC) for which the cloud chamber will be unstable with respect to these convective disturbances. Having to determine these conditions empirically for each investigation is a serious limitation to the applicability of the TDCC. In addition, the onset of such disturbances may be subtle, making it all but impossible to detect visually. Furthermore, while repeatedly solving Eqs. (1) and (2) will provide the necessary information to predict the (possible) onset of unstable behavior, it will be helpful to investigators using (or wishing to use) the TDCC to have a simple, reliable method to predict the conditions that could give rise to unstable behavior. We now provide just such a method.

The total pressure at which the total density profile along the wall first becomes vertical (e.g., our defined limit of stable operation or stability limit) at the upper plate can be estimated using the following equation (refer to the Appendix for details of the derivation):

$$
p_{\text {tot, } \lim }=\left(\frac{M_{v}}{M_{g}}-1\right) \cdot\left(\frac{B}{T_{U}}-1\right) \cdot p_{\text {vap }}\left(T_{U}\right),
$$

where $M_{v}$ and $M_{g}$ are the molecular weights of the condensable vapor and the background gas, respectively; $B$ is a constant in the Clausius-Clapeyron vapor pressure equation; $T_{U}$ is the upper plate surface temperature; and $p_{\text {vap }}$ is the equilibrium vapor pressure of the condensable at the upper plate temperature. For pressures below this stability limit, the density gradient is stable throughout the chamber and convective flow cannot develop. This pressure represents the upper limit of the total pressure for proper chamber operation. At pressures above this limit, density minima occur and convective flows such as those we have described can develop. We emphasize that this type of convective instability is expected to be subtle and difficult to detect visually. In fact, the chamber will seem to be operating perfectly, and the results obtained are reproducible and appear reasonable. However, the presence of such convective flows can decrease or increase the actual nucleation rate by transporting less or additional material into the nucleation zone. If, for example, the former is the case, then during critical supersaturation measurements, the temperature difference between the plates would have to be increased to compensate for this convective flow, and the calculated supersaturation will be too large. For the first time, Eq. (3) provides a criterion that can be used to easily determine, in a semiquantitative fashion, appropriate operating ranges for using a TDCC.

We have reexamined all the total pressure-dependent nucleation data reported from our laboratory ${ }^{2,5,6}$ in the context of this stability limit. Figure 11 shows a stability plot for the system 1-propanol in hydrogen. The solid line represents the stability limit as computed using Eq. (3); it separates the stable region (without density minima) from the unstable region (density minima will occur near the wall). Note that the stability limit curve increases sharply due to the exponential dependence of the vapor pressure on temperature. We conclude that it is preferable to operate the TDCC at high temperatures, where the accessible pressure range is large. This is consistent with the conclusion stated earlier, based upon the solution of Eqs. (1) and (2), that stable TDCC operation 


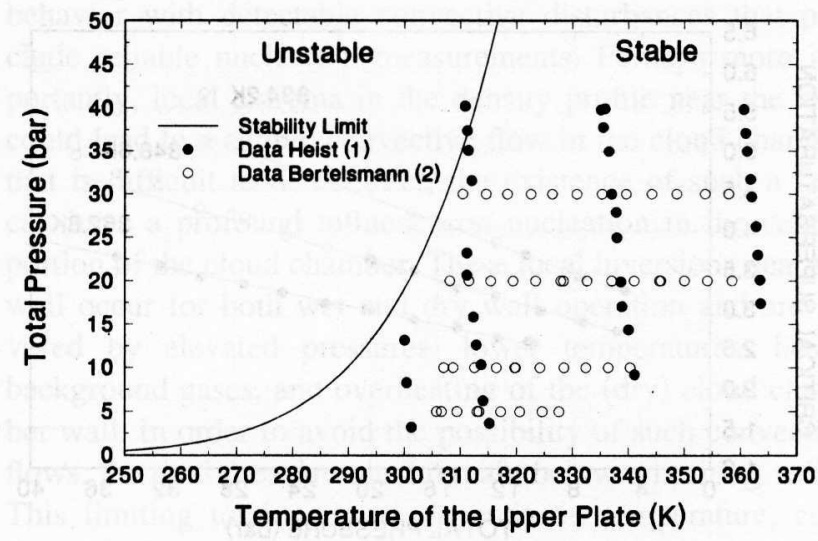

FIG. 11. Plot of the total pressure versus the upper plate temperature, indicating the region of stable TDCC operation for 1-propanol in hydrogen. The data represented by solid circles was taken from Ref. 5 , and the data represented by open circles was taken from Ref. 2 .

is enhanced at higher temperatures. It is also consistent with observations reported by Fisk et al. ${ }^{4}$ We also note from Fig. 11 that at low temperatures, density minima, and convective flow can occur at pressures as low as 1 bar or less. This is also consistent with our earlier conclusions. It is important to emphasize that when operating the chamber in the (potentially) unstable region, results obtained from nucleation rate or critical supersaturation measurements are no longer reliable, even though conditions inside the chamber (temperature, pressure, nucleation rate) appear stable and the results are reproducible. Furthermore it is important to note that this stability limit curve was computed under the assumption of no wall overheating. If the chamber wall is heated using heating wires, overheating will occur and can easily lead to density minima and possible convective flow below the pressure limit computed using Eq. (3).

In Fig. 11, data from our laboratory for the 1-propanol/ hydrogen system is shown as solid circles. The position of these circles is determined using the (measured) total pressure and the (measured) upper plate temperature. Each of the four vertical rows of circles corresponds to experiments in which the total pressure was varied at a constant nucleation temperature of $319,334,348$, and $363 \mathrm{~K}$, respectively. In Fig. 11, all but one data point lie within the stable region. We point out that wall heating was not used in these experiments. The location of the data points in Fig. 11 is entirely consistent with the stable behavior of the density profiles plotted for 1-propanol in hydrogen at $363 \mathrm{~K}$ in Fig. 10 [obtained through the solution of Eqs. (1) and (2)]. Thus, the pressure dependence of the critical supersaturation that we reported earlier cannot be attributed to instability and convective flow arising from extrema in the density profile in the HPCC. We have obtained similar results with all our data for the systems of methanol, ethanol, and 2-propanol in hydrogen and helium.

The use of heavier background gases and/or lower vapor pressure condensable vapors causes the stability limit curve to shift to higher temperatures or to lower pressures. This is indicated in Figs. 12 and 14. Figure 12 shows the stability

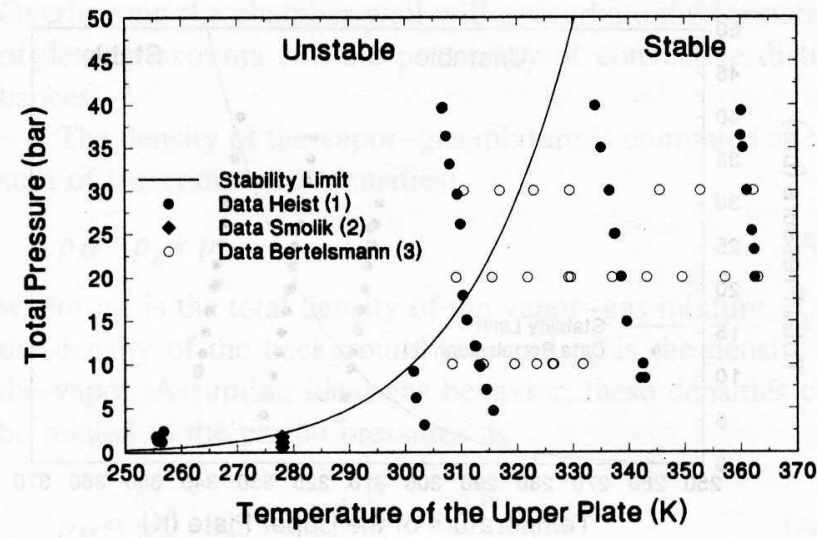

FIG. 12. Plot of the total pressure versus the upper plate temperature, indicating the region of stable TDCC operation for 1-propanol in helium. The data represented by solid circles was taken from Ref. 5 , the data represented by open circles from Ref. 2 , and the data represented by solid diamonds from Ref. 7 .

limit for the system 1-propanol in helium. The solid stability curve is shifted to the right, and we note that several of our data points lie within the unstable region. These points correspond to experiments performed at varying total pressure and a constant nucleation temperature of $334 \mathrm{~K}^{5}$ Density gradients for several of these experiments are shown in Fig. 9 [obtained by the solution of Eqs. (1) and (2)]; the existence of density minima for pressures above 20 bar predicted using Eq. (3) is verified in Fig. 9. Our previous discussion suggests that convective flow may have developed in those experiments, which could have led to a reduction in rate. If so, then in order to maintain the rate at approximately 2-4 drops $/ \mathrm{cm}^{3} / \mathrm{s}$, the temperature difference between the plates would have to be increased, leading to higher calculated supersaturations. Figure 13 shows the dependence of the critical supersaturation on total pressure for this system. In this figure, we have excluded the unstable data points that penetrate into the unstable region in Fig. 12 from the regression analysis and determined the slope of the pressure dependence using only data points up to 20 bar. As can be seen in Fig. 13 , the data points obtained for a nucleation temperature of $334 \mathrm{~K}$ and pressure above 20 bar lie above the line, which is

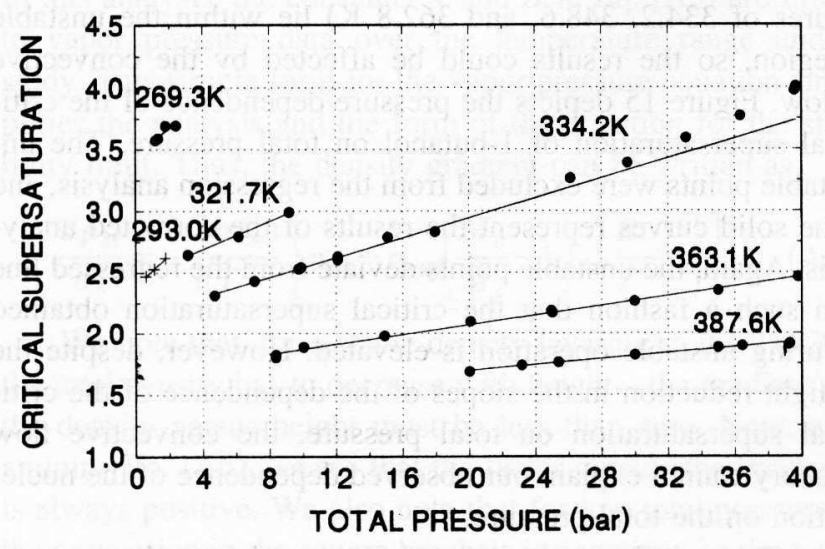

FIG. 13. Plot of the critical supersaturation versus the total pressure for 1-propanol in helium. The data shown in this plot corresponds to the data shown in Fig. 12. 


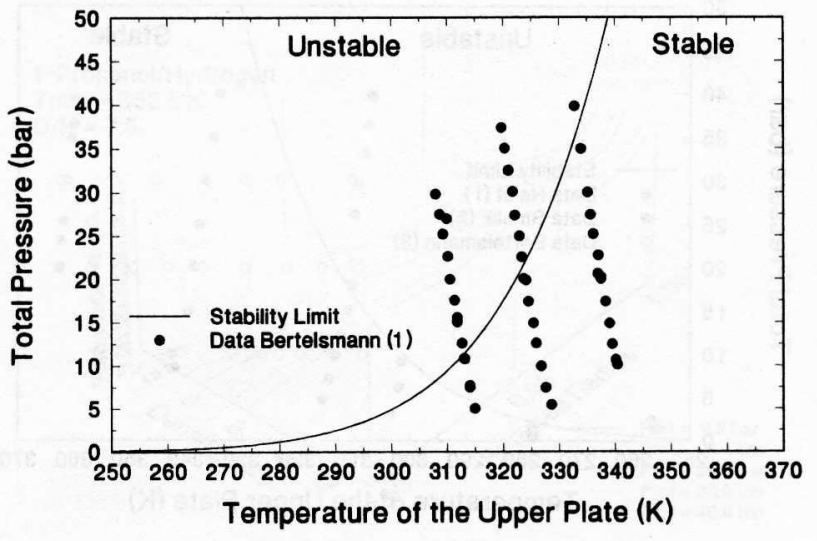

FIG. 14. Plot of the total pressure versus the upper plate temperature, indicating the region of stable TDCC operation for 1-butanol in helium. The data represented by solid circles was taken from Ref. 2 .

to be expected if the convective flow resulted in a lowering of the nucleation rate. The existence of the convective flow explains the tendency of some high-pressure data points to "curve upward," a behavior that we observed but could not explain before. It is important to reinforce our observation that during these experiments, the operation of the chamber appeared normal, and the data were reproducible (as can be seen for two experiments at approximately 40 bar). ${ }^{5}$ Only our two-dimensional analysis of the density profiles (performed afterward) suggested the possibility of a subtle, convective flow, which could adversely affect our results. The exclusion of the unstable data points from the regression lead to a minor reduction in the slope of the pressure dependence of the critical supersaturation. However, we must emphasize that instability and convective flow cannot account for our observed dependence of nucleation on total pressure.

We have carried out a similar analysis for the system 1-butanol in helium. 1-butanol has a lower vapor pressure than 1-propanol, so the stability limit is shifted to higher temperatures or lower pressures. The stability limit curve for 1-butanol in helium is shown in Fig. 14. A number of the data points taken in our laboratory (at nucleation temperatures of $334.2,348.6$, and $362.8 \mathrm{~K}$ ) lie within the unstable region, so the results could be affected by the convective flow. Figure 15 depicts the pressure dependence of the critical supersaturation of 1-butanol on total pressure. The unstable points were excluded from the regression analysis, and the solid curves represent the results of the corrected analysis. Again, the unstable points deviate from the regressed line in such a fashion that the critical supersaturation obtained during unstable operation is elevated. However, despite the slight reduction in the slopes of the dependence of the critical supersaturation on total pressure, the convective flow theory cannot explain our observed dependence of the nucleation on the total pressure.

The stability analysis for nitrogen and argon background gases shows a stability limit that is shifted toward higher pressures and lower temperatures. The limit of a stability curve for 1-propanol in nitrogen is shown in Fig. 16. In this

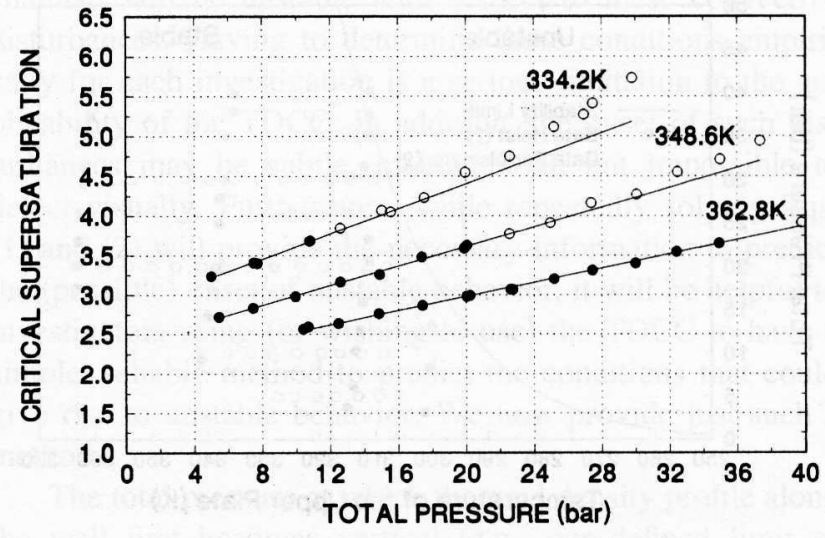

FIG. 15. Plot of the critical supersaturation versus the total pressure for 1-butanol in helium. The data shown in this plot corresponds to the data shown in Fig. 14.

case all of the data points taken in our laboratory lie in the unstable region. Thus, the reported pressure effect for 1-propanol in nitrogen (and similarly for 1-butanol in nitrogen) is likely to be exaggerated (but not necessarily eliminated) due to convective flow. ${ }^{2}$ Based on our observations for the 1-propanol and 1-butanol in hydrogen and helium systems, there is no reason to doubt the existence of a pressure effect in these systems, as well. However, the results obtained with the HPCC cannot be used to access the magnitude of the effect.

\section{SUMMARY}

In this paper we have addressed the operational stability of the vapor-gas mixture in the TDCC with respect to buoyancy-driven convective flow disturbances. We examined the conditions for stable operation in the central portion of the TDCC, as well as conditions necessary for stable operation in the vicinity of the chamber wall. The density profile in the central portion of the TDCC has to decrease monotonically with height since even local inversions can propagate through the chamber and cause overall unstable

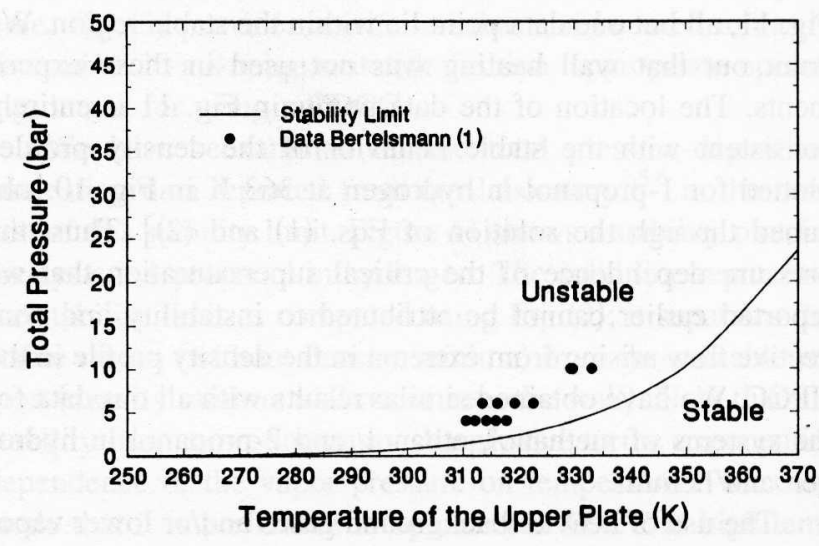

FIG. 16. Plot of the total pressure versus the upper plate temperature, indicating the region of stable TDCC operation for 1-propanol in nitrogen. The data represented by solid circles was taken from Ref. 2 . 
behavior with detectable convective disturbances that preclude reliable nucleation measurements. Perhaps more importantly, local extrema in the density profile near the wall could lead to a subtle, convective flow in the cloud chamber that is difficult to detect. Yet, the existence of such a flow can have a profound influence on nucleation in the central portion of the cloud chamber. These local inversions near the wall occur for both wet and dry wall operation and are favored by elevated pressures, lower temperatures, heavy background gases, and overheating of the (dry) cloud chamber wall. In order to avoid the possibility of such convective flows, the total pressure must remain below a limiting value. This limiting total pressure depends on temperature, condensable vapor, and background gas.

We have developed a simple method to estimate the limiting total pressure in a TDCC that will support stable operation. From results of our investigations based upon this method, it appears that the TDCC is best suited for experiments at higher temperatures, where the accessible total pressure range is largest. For (relatively) low vapor pressure materials, such as 1-pentanol or other high molecular weight alcohols and alkanes, stability limitations may preclude making reliable nucleation measurements at low temperatures altogether. Using Eq. (3) provides a quick, simple way to determine the feasibility of TDCC (and HPCC) experiments prior to actual experiments.

All our two-dimensional transport calculations [Eqs. (1) and (2)] and limit of stability calculations [Eq. (3)] have involved the lower molecular weight alcohols since those are the fluids studied most extensively in our laboratory. Extension of these model calculations to other systems is necessary, and we are currently making progress in that direction.

\section{ACKNOWLEDGMENTS}

We would like to acknowledge the helpful discussions with John Ofori, Peng Ye, Shenbo Yu, and Donald He and support by the Department of Chemical Engineering at the University of Rochester.

\section{APPENDIX}

\section{Stability limit for operation of a diffusion cloud chamber}

For proper operation of the diffusion cloud chamber, the total density profile must decrease with height throughout the TDCC. Density minima are most likely to occur at the wall where the condensable is depleted (wet wall) or the temperature elevated (dry wall). In the following, we derive a semiquantitative equation that can be used to predict the total pressure (as a function of temperature) that defines the conditions under which density minima at the wall can occur. Experiments performed at pressures above this limit are susceptible to a subtle convective flows, which cannot be easily detected yet may significantly affect cloud chamber performance and critical supersaturation and nucleation rate data. The analysis presented below is valid for either a wet or a dry wall, but does not allow for overheating at the wall.
Overheating the chamber wall will exacerbate the formation of density extrema and the possibility of convective disturbances.

The density of the vapor-gas mixture is computed as the sum of the component densities:

$$
\rho_{M}=\rho_{g}+\rho_{v},
$$

where $\rho_{M}$ is the total density of the vapor-gas mixture, $\rho_{g}$ is the density of the background gas, and $\rho_{v}$ is the density of the vapor. Assuming ideal gas behavior, these densities can be related to the partial pressures as

$$
\rho_{M}=\frac{M_{g}}{R T}\left[p_{\text {tot }}+p_{\text {vap }}\left(\frac{M_{v}}{M_{g}}-1\right)\right],
$$

where $M_{g}$ and $M_{v}$ are the molecular weights of the background gas and condensable vapor, respectively; $p_{\text {tot }}$ and $p_{\text {vap }}$ are the total pressure and the equilibrium vapor pressure, respectively; and $R$ is the gas constant and $T$ the temperature. At the wall, the partial pressure of the condensable is given by its vapor pressure (assuming the wall is not overheated). The derivative of the density with respect to height, $z$, is given by

$$
\begin{aligned}
\frac{d \rho_{M}}{d z}= & -\frac{M_{g}}{R T^{2}}\left[p_{\text {tot }}+p_{\text {vap }}\left(\frac{M_{v}}{M_{g}}-1\right)\right] \frac{d T}{d z}+\frac{M_{g}}{R T}\left(\frac{M_{v}}{M_{g}}-1\right) \\
& \times \frac{d p_{\text {vap }}}{d T} \frac{d T}{d z}
\end{aligned}
$$

We assume that the temperature profile decreases monotonically with height (which may not be true in the case of overheating at the wall); it does not, however, have to be linear. It can be expressed as

$$
\frac{d T}{d z}=-\alpha(z), \quad \alpha>0 .
$$

For relatively small ranges of temperatures we use a Clausius-Clapeyron form for the equilibrium vapor pressure:

$$
p_{\text {vap }}(T)=p_{c} \exp (A-B / T) .
$$

In this analysis, the constants $A$ and $B$ in Eq. (A5) are fitted to vapor pressure data over the temperature range under study. This simple form for the vapor pressure equation simplifies the analysis and the form of the equation for the stability limit. Thus, the density gradient can be written as

$$
\frac{d \rho_{M}}{d z}=\frac{M_{g} \alpha(z)}{R T^{2}}\left[p_{\text {tot }}-p_{\text {vap }}\left(\frac{M_{v}}{M_{g}}-1\right) \cdot\left(\frac{B}{T}-1\right)\right] .
$$

We note that, for a stable density layering in the TDCC, the total density has to decrease with height-the gradient of the density versus height must be less than zero. Now, examining Eq. (A6), we see that the factor outside the brackets is always positive. We also note that for low total pressures, the expression in the square brackets is negative. As the total pressure increases, it begins to dominate the terms in the square brackets and leads to a (local) positive gradient. A minimum in density occurs when the gradient vanishes, i.e. 
the term in brackets becomes zero. As our two-dimensional model calculations have shown, this tends to occur first at the upper plate. Thus, the temperature of the upper plate may be used to compute the total pressure at which the density first develops a minimum. The stability limit for the operation of the diffusion cloud chamber is given by

$$
p_{\text {tot, } \lim }=\left(\frac{M_{v}}{M_{g}}-1\right) \cdot\left(\frac{B}{T_{U}}-1\right) \cdot p_{\text {vap }}\left(T_{U}\right) .
$$

For total pressures below that computed using Eq. (A7), the density gradient is stable throughout the chamber, and conditions supporting convective flow do not exist. This is the range of total pressure in which the TDCC should be operated. For total pressures equal or slightly larger than the limit computed by this equation, density minima occur, however, they may be too small or too close to the wall for convective flow to develop. For pressures significantly larger than the stability limit, our experimental data suggest that a disturbance such as a convective flow is likely to develop. Results obtained in this range, even though reproducible, are not reliable and should be suspect.

\section{LIST OF SYMBOLS}

$\begin{array}{ll}r & \text { radial coordinate } \\ z & \text { axial coordinate } \\ x & \text { mole fraction of the condensable in the gas phase } \\ T & \text { temperature } \\ c & \text { concentration } \\ D_{A B} & \text { binary diffusion coefficient }\end{array}$

$b \quad$ exponent for the temperature dependence of the diffusion coefficient

$c_{p} \quad$ heat capacity of the vapor

$\lambda$ thermal conductivity

\section{Stability limit calculation (Appendix)}

$T_{U} \quad$ temperature of the upper plate

$P_{\text {tot }} \quad$ total pressure

$P_{\text {vap }} \quad$ equilibrium vapor pressure

$P_{c} \quad$ critical pressure of the condensable

$\rho_{M} \quad$ density of the vapor-gas mixture

$\rho_{v} \quad$ density of the condensable vapor

$\rho_{g} \quad$ density of the background gas

$M_{v} \quad$ molecular weight of the condensable vapor

$M_{g} \quad$ molecular weight of the background gas

$A, B$ constants in the vapor pressure equation

$\alpha(z) \quad$ temperature gradient

\footnotetext{
${ }^{1}$ A. Bertelsmann and R. H. Heist, J. Chem. Phys. 106, 610 (1997), preceding paper.

${ }^{2}$ A. Bertelsmann, R. Stuczynski, and R. H. Heist, J. Phys. Chem. 100, 9762 (1996).

${ }^{3}$ Reported at Nucleation Experiments: State of the Art and Future Developments, an International Workshop Held at the Czech Technical University, Prague, Czech Republic, June, 1995.

${ }^{4}$ J. A. Fisk, V. M. Chakarov, and J. L. Katz, J. Chem. Phys. 104, 8657 (1996).

${ }^{5}$ R. H. Heist, M. Janjua, and J. Ahmed, J. Phys. Chem. 98, 4443 (1994)

${ }^{6}$ R. H. Heist, M. Janjua, and J. Ahmed, J. Phys. Chem. 99, 375 (1995).

${ }^{7}$ J. Smolik, data presented at the European Aerosol Conference, Helsinki, Finland, 1995.
} 